\title{
Affinités électives et charité interprétative
}

Monsieur Miguel Vale de Almeida, Luiz Ferraz, Madame Christine Ritui

\section{Citer ce document / Cite this document :}

Vale de Almeida Miguel, Ferraz Luiz, Ritui Christine. Affinités électives et charité interprétative. In: Recherches en anthropologie au Portugal, $\mathrm{n}^{\circ} 1,2004$. Itinéraire d'un anthropologue : João de Pina Cabral. pp. 207-211;

doi : 10.3406/rap.2004.1221

http://www.persee.fr/doc/rap_1240-3474_2004_num_10_1_1221

Document généré le 25/04/2016 


\section{Affinités électives et charité interprétative}

Miguel VAIE DI: ALALIISA

"()f course, what was in their minds, we will nerer be able to knon. But, through a process of interpretive charity, I think we managed to get a fairly good notion of what might balle been at stake" (Pina Cabral, Albinos don't die, 20(02).

"We don't get to know people when they come to us; we bave to go to them so as to learn what they are like " (Goethe, Elective Affinities, 1809).

Pina (abral affirme, dans un entretien publié dans ce volume, que dans les années 1990 est née une nouvelle anthropologie au Portugal. Cette affirmation se rattache directement à un optimisme qui envisage l'anthropologie comme une discipline d'avenir, et à un pessimisme - voire à une prudence - qui reconnait la faiblesse de la discipline sur le plan de la reconnaissance scientifique et du financement au niveau européen.

Il y a dans ce raisonnement quelque chose qui ressemble aux affirmations entendues dans des domaines normalement catalogués comme appartenant aux humanités ou même au domaine artistique: le processus créatif propre aux formes critiques et autocritiques de la compréhension du monde - surtout si elles s'appuient sur l'engagement et le travail ordinairement individuels - conduit au surgissement cyclique de nouvelles formes de voir, de faire, d'interpréter et d'analyser. Ces formes rendent compte du 
monde d'une manière que l'on devine utile et plus proche du réel, mais c'est aussi cette caractéristique qui rend difficile l'institutionnalisation, la formation d'un champ solide, la mise en place de routines, la création d'attentes utilitaires de la part des pouvoirs, conduisant ainsi à une sorte d'entrave à l'acceptation et au financement, aussi bien qu'à des entraves à l'expansion et à la création d'une masse critique : "Votre travail est excellent, je lis toujours vos livres, mais malheureusement vos projets ne cadrent pas avec nos priorités."

Normalement, nous acceptons cet état des choses lorsque nous situons l'anthropologie dans le domaine des humanités ou lorsque nous acceptons certaines condamnations à mort qui expliquent la perte de pouvoir de l'anthropologie par la perte d'un supposé objet d'étude. Effectivement, des évaluations de ces deux genres coinncident avec deux effets d'hégémonie apparus dans les sciences, dans leur rapport à la société et aux États. I a première affirmation découle d'un jugement de valeur qui privilégie l'utilitarisme et la technicité - pour ne pas dire la rentabilité - en établissant une (fausse) dichotomie entre les sciences technologiques et les sciences humaines et les arts ; la deuxième appartient à une (fausse) interprétation de l'Histoire qui, pour être vraie, devrait admettre que la sociologie a perdu son objet d'étude - si, par l'absurde, celui-ci se situait dans le contexte historique d'un Comte ou d'un Durkheim.

Ces effets d'hégémonie sont intensifiés au Portugal par le fait que l'aire sociologique a, jusqu'à un certain point, intériorisé cette hégémonie, se présentant comme un savoir utilitaire et technique, situation qui possède un effet double et perturbateur: si, d'un côté, elle a besoin d'écarter les disciplines-sceurs vers les marges humanistiques, de l'autre, la sociologie n'est jamais acceptée de plein droit au sein de l'hégémonie technocratique et néo-libérale.

Pina Cabral appartient à une "génération " ou à un réseau d'anthropologues (ou encore pour être plus exact à une "affinité élective ») à laquelle il me plaît de m'identifier. Il s'agit d'un groupe diffus dont les affinités, entre autres, peuvent être ainsi énumérées: ce groupe croit que l'objet de l'anthropologie n'est pas mort car il ne peut être confondu avec les "primitifs» colonisés; que l'anthropologie produit un savoir communiquant avec - mais différencié de - la sociologie; que l'anthropologie ne se limite ni à la production nord-américaine, ni à une "réserve ethnologique 
nationale ", mais est plutôt de nature globale et à plusieurs facettes (l'une des affirmations de cette prise de position est, peut-être, la participation à la construction de l'EASA); et il croit à une anthropologie qui a intériorisé un éventail d'expériences épistémologiques, méthodologiques et politiques aussi diversifiées, qui a fourni à la discipline des outils qui lui permettent de rendre compte, simultanément, des structurations sociales et des multiples sens créés par les expériences sociales à l'époque contemporaine.

Des affirmations de cet ordre ne peuvent se faire qu'après qu'a été considérée la validité des raisonnements, de sens contraire, qui l'induisent comme réaction. Nous connaissons tous par cour l'histoire des nouveaux terrains anthropologiques à la période postéricure aux décolonisations. Nous connaissons tous l'histoire de l'autocritique de l'anthropologie, de sa méthode, de son écriture, de sa politique. Nous connaissons tous l'histoire de la rencontre avec la réflexivité anthropologique. Nous les connaissons, au moins, au niveau international, mais combien de fois sont-ils simplement confondus avec les contextes anglo-américains? Qu'en est-il de notre contexte?

Le travail de Pina Cabral est en lui-même, j'en suis sûr, comme une narration de cette Histoire. Voyons. D'abord, la biographie - celle strictement personnelle et celle académique, si intimement liées - qui le conduit au Mozambique, en $\Lambda$ frique du Sud, au Royaume-Uni, au Portugal, dans un parcours qui, pour simplifier, commence par un des empires coloniaux portugais et se poursuit dans le post-colonialisme des territoires de l'expansion historique de l'État portugais (dans l'expression par lui consacrée). C'est un parcours critique fait de marginalités inattendues : le jeune colon vit dans une ambiance en marge de la domination lusitanienne en $A$ frique, doute du missionnarisme anglican dans lequel il a été élevé, et partage les idées d'indépendance africaine. Dans le pays de l'apartheid, il étudie justement dans un des milieux le plus férocement anti-apartheid. In Angleterre, il assiste à la reformulation des champs anthropologiques classiques. It au Portugal, il découvre son pays d'origine comme un étranger. Tous ces éléments sont simultanément l'histoire d'un contexte de relations et l'histoire d'un parcours d'outsider inside et d'insider outside, qui constitue idéalement un anthropologue.

Ensuite, les thèmes et les terrains. En syntonie avec l'ouverture, la croissance et la diversification de l'anthropologie internationale - et la caracté- 
ristique centrale de Pina Cabral pourrait se définir comme "cosmopolitisme "-, cet anthropologue aborde la mort, la famille, le genre, la sexualité, l'ethnicité, le colonialisme et le post-colonialisme. Portugal, Macao, Mozambique, Brésil, ses terrains et centres d'intérêt sont des territoires de croisements historiques et sumboliques, qui renvoient à la construction d'un monde commun où, malgré tout, il est possible de penser ensemble - ne scrait-ce qu’à travers les "compatibilités équivoques» surgies dans les confrontations et dialogues de personnes situćes dans des marges multiples.

Infin, la capacité hỵbridisante de Pina Cabral. Il n'y a pas, dans son parcours, ces choix de modes et de modèles rigides, sectaires ou de chapelles, si fréquents dans certaines formes de fonctionnement de l'académie. Lit pourtant son éclectisme n'est pas synonyme - bien au contraire - de manque d'attention ou de profondeur, car la profondeur n'est pas incompatible avec l'extension. Ici encore le cosmopolite va de concert avec le flâneur, le voyageur, le romancier et l'homme préoccupé par l'éthique et la politique. C'est ainsi que nous le voyons écrire et produire, en portugais et en anglais, publiant avec une régularité étonnante; c'est ainsi que nous le voyons faire du terrain, au lieu de se livrer à la tranquillité de sa chaire; c'est ainsi que nous le voyons administrer, encourager, gérer, à l'échelle de l'EASA comme à celle des institutions où il travaille, passant par la création de think tanks, de séminaires et formations avancées, au lieu de se réfugier seulement dans sa production intellectuelle personnelle. Je suis sûr que c'est au cours des discussions ouvertes avec des collègues, disciples et informateurs - parfois avec de naturels équivoques compatibles, plutôt qu'avec des compatibilités équivoques - que se justifiait l'adoption de la "charité interprétative » de Davidson, ce "mouvement " éthique qui, non seulement sur le terrain, mais surtout avec ses collègues, engendre les affinités électives.

l a solidité de ses connaissances théoriques, des classiques de l'anthropologie et des ethnographies, n'empêche pas mais plutôt stimule le désir du nouveau - d'où l'encouragement à tant de recherches envisageant des problématiques et des terrains nouveaux. Dans le fond, Pina Cabral sait, comme il le dit si bien, que "le centre est une fantasmagorie" - une sorte de point de fuite observable seulement à travers la constante comparaison ethnographique, à travers une position "processuclle ", à travers une attention à l'action qui découle et arrive de l'interrelation sociale que constituent les personnes, les identités, les socialités - oui, bien sûr, ce vieux fantasme, 
la société.

I.e travail de Pina Cabral symbolise et synthétise ce que beaucoup d'entre nous essayons de faire avec l'anthropologie pratiquée ici : appréhender la réalité portugaise au-delà d'un paradigme spécifiquement ethnologique, et appréhender le monde post-colonial et globalisé au-delà d'un paradigme crypto-colonialiste. C'est dans ce parcours que j'identifie les affinités électives pour une nouvelle anthropologie au Portugal : cosmopolite, hybridisante, universelle, capable de rendre compte des formes multiples de la façon dont nous vivons (dans) un même monde.

Traduit du portugais par J,uiz FI:RRA\% et Christine RITUI 